\title{
Investigation of the deformation stability in the incremental sheet forming process
}

\author{
S. $\mathrm{Ai}^{1}$, B. $\mathrm{Lu}^{1,2, \mathrm{a}}, \mathrm{H} . \mathrm{Long}^{2}$, J. Chen ${ }^{1}$, and H. $\mathrm{Ou}^{3}$
}

${ }^{1}$ Department of Plasticity Technology, Shanghai Jiao Tong University, Shanghai 200030, China

${ }^{2}$ Department of Mechanical Engineering, University of Sheffield, Sheffield S1 3JD, UK

${ }^{3}$ Department of Mechanical, Materials and Manufacturing Engineering, University of Nottingham, Nottingham NG7 2RD, UK

\begin{abstract}
Incremental sheet forming (ISF) is a highly versatile and flexible process for rapid manufacturing of complex sheet metal parts. One of the unique characters of the ISF process is the improved formability comparing to conventional sheet forming process. This may be due to the localized deformation nature, which increases the deformation stability in the ISF process. Although many hypotheses have been proposed, there is no direct modelling and calculation of the ISF deformation stability. Aiming to obtain a better understanding of the ISF process, an analytical model was developed to investigate and analyse the material deformation stability in this work. Based on the analytical evaluation of stress variations and force equilibrium, a mathematical relationship between the maximum forming angle and the process stability condition was established. To validate the developed model, experiments were carried out by forming a hyperbolic part made of AA1100 material. The maximum forming angle, as an indicator to the ISF formability, was employed compare the analytical evaluation and experimental result. It was found that the ISF deformation stability is one of the key factors that affect the ISF formability.
\end{abstract}

\section{Nomenclature}

$\sigma_{\varphi} \quad$ meridional stress

$\sigma_{\theta} \quad$ circumferential stress

$\sigma_{t} \quad$ thickness stress

$\sigma_{s} \quad$ yield stress

$F_{\varphi} \quad$ meridional force

$F_{\theta} \quad$ tangential force

$\beta \quad$ forming angle

$t_{B} \quad$ sheet thickness in region $\mathrm{B}$ $\alpha \quad$ forming angle

$t_{0} \quad$ initial sheet thickness

$t \quad$ actual sheet thickness $\backslash$

$r_{t}$ tool radius

$F_{t}$ force in thickness direction

$\theta_{0} \quad$ contact angle in the tangential direction

$S$ the area of the boundary between region A and B

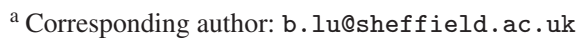

This is an Open Access article distributed under the terms of the Creative Commons Attribution License 4.0, which permits unrestricted use, distribution, and reproduction in any medium, provided the original work is properly cited. 


\section{MATEC Web of Conferences}

\section{Introduction}

Incremental sheet forming (ISF) is a flexible process for manufacturing of small-batched and customized sheet metal products. Compared with conventional sheet forming technologies, ISF does not require specified forming tools, which reduced cost and lead time in manufacturing process. In the investigation of the ISF process, many technologies such as two point incremental forming(TPIF) [1], double side incremental forming(DSIF) [2] have been proposed and many efforts have been made for improved ISF technology during the past years [3-5].

One unique characterization of the ISF process is the increased formality. This high ISF formability has been investigated with many theories proposed. Emmens and Boogaard [6] found that the effect of continuous bending under tension is a critical factor to the increased formability. Ackson and Allwood [7] demonstrated that the material deformation of ISF is due to the combination of bending, stretching and shearing. Allwood and Shoulder [8] suggested that the through thickness shear is a significant factor to increase the sheet formability. Eyckens et al. [9] investigated the through thickness shear effect by using the Marciniak-Kuczynski model and suggested that this effect would enhance the formability. Eyckens et al. [10] also argued that the dominant material deformation mechanism (i.e. shearing or bending) depends on the specific process conditions. Concerning the ISF modelling, Silva et al. [11] presented an analytical model based on membrane approach to address the mechanism of ISF process and the effect of forming parameters on the ISF formability. Fang et al. [12] established an analytical model to analyse the effects of bending and material hardening on the SPIF formability. In a recent research, Lu et al. [13] investigated the effect of friction on the sheet deformation and fracture behaviour in the ISF process. The above published literatures identified that the major SPIF deformation mechanism is the combination of bending, stretching and shearing deformation depending on process conditions.

Aiming to obtain a better understanding of the ISF process, an analytical model has been developed in this work. Focusing on the deformation stability, analytical evaluation of stress variations have been implemented and the process stability condition is established. To validate the developed model, experiments are carried out by forming a hyperbolic part. The results are examined and the mechanism of the ISF deformation stability is discussed to further enhance the understanding of the ISF process.

\section{Theoretical investigation}

During the incremental sheet forming process, a localized deformation zone exists. Deformation mainly occurs in and near the contact area, while other regions don't involve any plastic deformation. Based on this deformation feature, a theoretical model has been presented as shown in Fig. 1. Two regions, including the contact region $\mathrm{A}$ and the neighbouring region $\mathrm{B}$ in the inclined wall are considered in the model. This model is based on following assumptions:

(1) Plane strain occurs in most of the deformation zone, while the biaxial stretching deformation at the ISF part corner is not considered in this work.

(2) Friction and shear effects are ignored with delicate lubrication.

In the region $\mathrm{A}$, considering the effect of stretching and bending, an equilibrium equation in the thickness direction can be established [12]:

$$
\left(\sigma_{t}^{A}+d \sigma_{t}^{A}\right) \cdot(r+d r) \cdot d \varphi-\sigma_{t}^{A} \cdot r \cdot d \varphi-\sigma_{\varphi} \cdot \sin \left(\frac{d \varphi}{2}\right) \cdot d r-\left(\sigma_{\varphi}+d \sigma_{\varphi}\right) \cdot \sin \left(\frac{d \varphi}{2}\right) \cdot d r=0 .
$$

With the consideration of the power hardening law $\sigma_{s}=K \cdot \bar{\varepsilon}^{n}$ and Mises yield criterion, Eq. (1) can be solved and the stress variation in the thickness direction can be obtained.

$$
\sigma_{t}^{A}=\frac{\frac{K}{\sqrt{3}}\left(\frac{2}{\sqrt{3}}\right)^{n}}{n+1}\left\{\left[\ln \frac{r \cdot t_{0}}{r_{t} \cdot t}\right]^{n+1}-\left[\ln \frac{\left(r_{t}+t\right) \cdot t_{0}}{r_{t} \cdot t}\right]^{n+1}\right\} .
$$




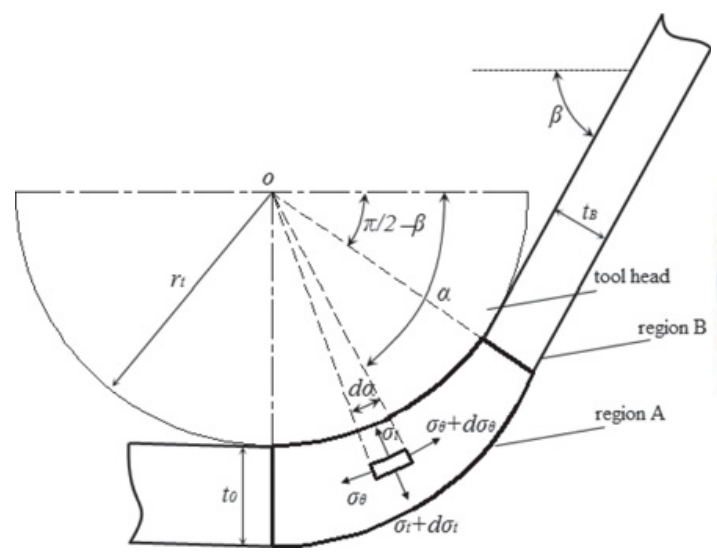

(a)

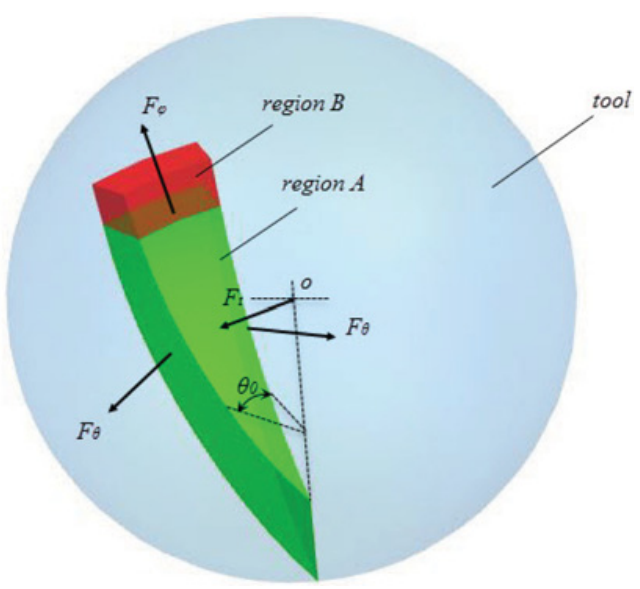

(b)

Figure 1. Model definition: (a) stress state; (b) force equilibrant.

Concerning the contact in the ISF process, the contact area can be estimated as a fan-shaped sector. Considering the contact area as a whole, it is subjected to the force balance in the all the principle directions. In the meridional and circumferential direction, the equilibrant equations can be given by Eqs. (3) and (4), respectively:

$$
\begin{gathered}
F_{\varphi}=F_{t} \cdot \sin \frac{\beta}{2}+2 F_{\theta} \cdot \sin \frac{\theta_{0}}{2} \cdot \cos \beta \\
F_{t} \cdot \cos \frac{\beta}{2}=2 F_{\theta} \cdot \sin \frac{\theta_{0}}{2} \cdot \sin \beta .
\end{gathered}
$$

After simplification, it can be obtained that

$$
F_{\varphi}=F_{t} \cdot\left(\sin \frac{\beta}{2}+\frac{\cos \beta}{2 \sin \frac{\beta}{2}}\right)=\int_{-\frac{\theta_{0}}{2}}^{\frac{\theta_{0}}{2}} \int_{\alpha_{0}}^{\frac{\pi}{2}}\left|\sigma_{t}\right| \cdot r_{t} \cdot d \alpha \cdot r_{t} \cdot \cos \alpha \cdot d \theta \cdot \cos \theta \cdot\left(\sin \frac{\beta}{2}+\frac{\cos \beta}{2 \sin \frac{\beta}{2}}\right) \cdot
$$

The force in the thickness direction $F_{t}$, namely the counteract force between the tool and the sheet can be considered as an integral of the stress in the thickness direction.

Under the plane strain condition, the ISF sheet thickness follows the sine law: $t=t_{0} \cdot \sin \alpha$. Combining Eqs. (2) and (5) with sine law, the force values in three directions can be obtained.

On the boundary between regions $\mathrm{A}$ and $\mathrm{B}$, according to force balance, it can be obtained that:

$$
F_{\varphi}=\sigma_{\varphi}^{B} \cdot S=\sigma_{\varphi}^{B} \cdot r_{t} \cdot \sin \beta \cdot 2 \sin \frac{\theta_{0}}{2} \cdot t_{B}
$$

In region $\mathrm{B}$, there is no contact between the forming tool and the sheet and the contact stress $\sigma_{t}=0$. With the consideration of plane strain condition, $\sigma_{\theta}=\sigma_{\varphi} / 2$ and Mises yield criterion, it can be obtained that:

$$
\sigma_{s}^{B}=\sqrt{\frac{3}{2} \sigma_{i j}^{\prime} \sigma_{i j}^{\prime}}=\frac{\sqrt{3}}{2} \sigma_{\varphi}^{B} .
$$

Under plane strain condition, $\varepsilon_{\varphi}=-\varepsilon_{t}=-\ln (\cos \beta)$ and $\varepsilon_{\theta}=0$. The equivalent strain can be obtained by: $\bar{\varepsilon}^{B}=\sqrt{\frac{2}{3} \varepsilon_{i j} \varepsilon_{i j}}=-\frac{2}{\sqrt{3}} \ln (\cos \beta)$. By further including the power hardening law, it can be 


\section{MATEC Web of Conferences}

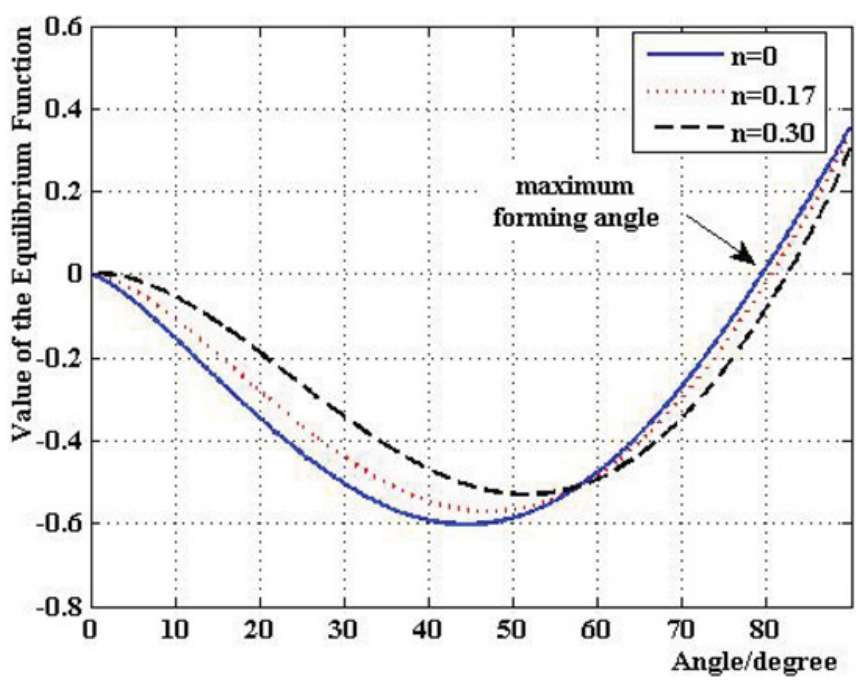

Figure 2. Illustration of the left part of the equation and the calculation of the maximum forming angle.

obtained that,

$$
\sigma_{s}^{B}=K \cdot\left(\frac{2}{\sqrt{3}} \ln \frac{1}{\cos \beta}\right)^{n} .
$$

Combining equation (7) and (8), the meridional stress component in region B can be obtained:

$$
\sigma_{\varphi}^{B}=\frac{2}{\sqrt{3}} K \cdot\left(\frac{2}{\sqrt{3}} \ln \frac{1}{\cos \beta}\right)^{n} .
$$

Substituting Eqs. (2), (5) and (9) into Eq. (6), it can be obtained that:

$$
\begin{aligned}
& f(\beta, n)=\int_{\cos \beta}^{1}\left\{\left[\ln \frac{t_{0}}{t_{0} \cdot \sin \alpha}\right]^{n+1}-\left[\ln \frac{\left(r_{t}+t\right) \cdot t_{0}}{r_{t} \cdot t_{0} \cdot \sin \alpha}\right]^{n+1}\right\} \cdot d(\sin \alpha) . \\
& \frac{1}{\sqrt{3}} \frac{r_{t}}{t_{0}} \cdot\left(\frac{2}{\sqrt{3}}\right)^{n} \cdot \frac{1}{n+1} \cdot\left(\sin \frac{\beta}{2}+\frac{\cos \beta}{2 \sin \frac{\beta}{2}}\right)-\frac{2}{\sqrt{3}}\left(\frac{2}{\sqrt{3}} \ln \frac{1}{\cos \beta}\right)^{n} \cdot \sin \beta \cdot \cos \beta=0 .
\end{aligned}
$$

By solving Eq. (10), the maximum forming angle can be calculated. In common manufacturing practices, the tool head with the radius of $5.0 \mathrm{~mm}$ and the original sheet thickness with the value of $1.0 \mathrm{~mm}$ are usually employed. For material AA1100 sheet, $n=0.17 \mathrm{Eq}$. (10) can be solved when it reaches zero as shown in Fig. 2. The corresponding maximum forming angle is $80.6^{\circ}$. The forming angles for another two different work-hardening exponents $n=0$ and $n=0.30$ have also been solved. As can be seen in Fig. 2, work hardening could lead to an increase of the forming angle.

\section{Experimental validation}

ISF experiments are conducted to examine the fracture of a hyperbolic part. The experiments are implemented on a traditional 3-Axis CNC machine with a tool whose radius is $5.0 \mathrm{~mm}$. AA 1100 aluminium sheet with a hardening index of $n=0.17$ is employed in this work. The original sheet 


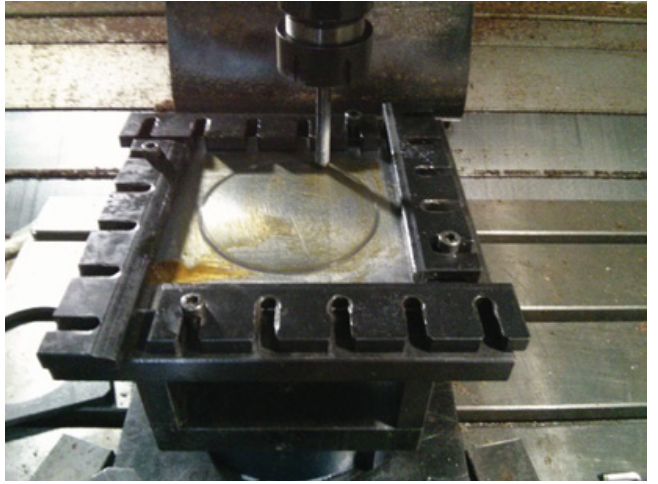

(a)

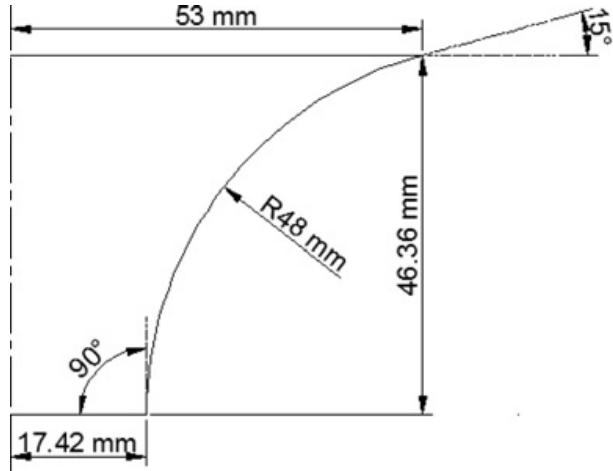

(b)

Figure 3. Configuration of the experimental set-up and designed part: (a) experimental setup; (b) designed part.

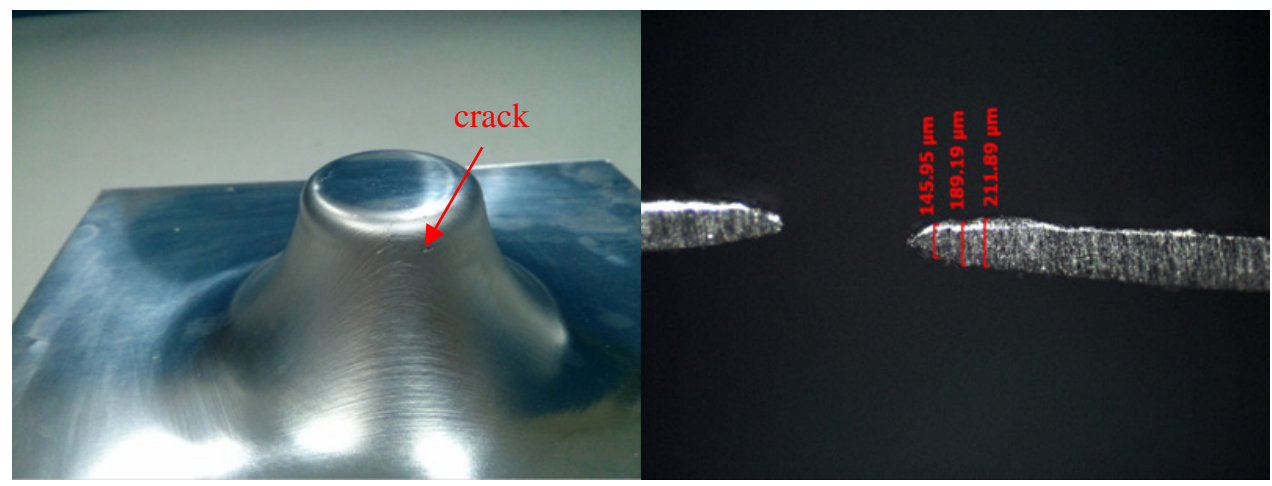

(a) (b)

Figure 4. Experimental results: (a) formed part; (b) macroscopic view of fracture location.

thickness is $1.0 \mathrm{~mm}$. In the experiment, the tool moves with a constant linear speed of $1200 \mathrm{~mm} / \mathrm{s}$. Solid lubricant MoS2 is employed to reduce the friction. The experimental set-up can be showed in Fig. 3.

The height of part is calculated according to the real-time Z-coordinate provided by the CNC machine. With the fracture height, the maximum forming angle can be obtained with the given CAD model. Three parts were made in the experiment and their fracture heights were recorded. For the truncated cone, the average fracture height is $37.4 \mathrm{~mm}$, which means the forming angle is $79.2^{\circ}$ based on the designed CAD model. Comparing with the theoretical value calculated in the previous section, there is only a small difference $(1.74 \%)$ between the two values.

Cross-sectional view of the fracture along meridional direction is presented in Fig. 4. Severe necking appears at the final stage of forming. It can be concluded for material AA1100 that the fracture in the ISF process is due to the instability of sheet deformation: with the increase of the forming angles, the part wall becomes thinner and thinner. On the other hand, with the increase of the forming angle, the forming force increases. At some stage, when the thin wall cannot support the high forming force, necking initializes and sheet fracture occurs.

Concerning the developed model, the analytical analysis in this work could predict the ISF fracture angle effectively with negligible computing time comparing with the conventional FEM and experimental approach. Furthermore, analytical analysis could provide a more fundamental explanation 


\section{MATEC Web of Conferences}

of the process. Forming parameters that affect the process can also be analysed with the model developed quantitatively. However, the developed model is only validated by a material with limited hardening index. Other materials with different properties may also be developed for further model validation in the future.

\section{Conclusions}

The conclusions of this work can be summarized as follows:

1. An analytical model with the consideration of bending effect has been developed to predict the fracture angle in the ISF process.

2. The developed model is validated by the ISF experiment of AA1100 material in forming a hyperbolic cone shape.

3. The mechanism of fracture of the AA1100 material in the ISF process is discussed and the deformation instability is the major cause of ISF fracture.

The authors are grateful for the support provided by the Marie Curie International Incoming Fellowship (628055 \& 913055) and International Research Staff Exchange Scheme (IRSES, MatProFuture project, 318968) within the 7th EC Framework Programme (FP7) and the Engineering and Physical Science Research Council of UK (EP/L02084X/1).

\section{References}

[1] S. Matsubara, J.S.T.P. 35 (1994)

[2] R. Malhotra, J. Cao, M. Beltran, D. Xu, J. Magargee, V. Kiridena, and Z.C. Xia, CIRP Annals Manu Tech. 61 (2012)

[3] T. Cao, B. Lu, D. Xu, H. Zhang, J. Chen, H. Long, and J. Cao, Int. J. Adv.Manu. Tech. 77 (2015)

[4] M.H. Zhang, B. Lu, J. Chen, H. Long, and H. Ou, Int. J. Adv. Manu. Tech. (2014)

[5] B. Lu, J. Chen, H. Ou, and J. Cao, J. Mat. Proc. Tech. 213 (2013)

[6] W.C. Emmens, A.H. Boogaard, J. Mat. Proc. Tech. 209 (2009)

[7] K. Jackson, J. Allwood, J. Mat. Proc. Tech. 209 (2009)

[8] J.M. Allwood, D.R. Shouler, Int. J. Plast. 25 (2009)

[9] P. Eyckens, A. Van Bael, and P. Van Houtte, Int. J. Plast. 25 (2009)

[10] P. Eyckens, B. Belkassem, C. Henrard, J. Gu, H. Sol, A. Habraken, J. Duflou, A. Bael, and P. Houtte, Int. J. Mat. Form. 4 (2011)

[11] M.B. Silva, M. Skjoedt, P.A.F. Martins, and N. Bay, Int. J. Mach. Tools. Manu. 48 (2008)

[12] Y. Fang, B. Lu, J. Chen, D.K. Xu, and H. Ou, J. Mat. Proc. Tech. 214 (2014).

[13] B. Lu, Y. Fang, D.K. Xu, J. Chen, H. Ou, N.H. Moser, and J. Cao, Int. J. Mach. Tools. Manu. 85 (2014) 